\title{
PINCHING THEOREMS OF SIMONS TYPE FOR COMPLETE MINIMAL SUBMANIFOLDS IN THE SPHERE
}

\author{
PAN YANGLIAN ${ }^{1}$
}

\begin{abstract}
In this paper the author proves some pinching theorems of Simons type for complete minimal submanifolds in the sphere, which generalize the relative results by Simons and Yau.
\end{abstract}

In this paper we first prove a pinching theorem for complete minimal submanifolds in the sphere, which has been proved by J. Simons [4] in the compact case. Namely, if $M^{n}$ is a complete submanifold in the sphere $S^{n+p}$, and if the square of the length of the second fundamental form of $M^{n}$ is not greater than $n /(2-1 / p)$, then $M^{n}$ is totally geodesic. When $p=1$, this theorem has been proved by $\mathrm{T}$. Hasanis [2].

Furthermore, it turns out that many pinching theorems in Yau's paper [5] can be proved to be still true for the complete case in the same way.

1. Local formulas and basic lemmas. Let $M^{n}$ be an $n$-dimensional manifold in the Euclidian unit sphere $S^{n+p}$. We choose a local field of orthonormal frames $e_{1}, \ldots, e_{n}, \ldots, e_{n+p}$ in $S^{n+p}$ such that, restricted to $M^{n}$, the vectors $e_{1}, \ldots, e_{n}$ are tangent to $M^{n}$. Let $w_{1}, \ldots, w_{n}$ be the field of dual frames. We shall make use of the following convention on the ranges of indices: $1 \leqslant i, j, k, \ldots \leqslant n, n+1 \leqslant$ $\alpha, \beta, \gamma, \ldots \leqslant n+p$; we shall agree that repeated indices are summed over the respective ranges.

We denote the second fundamental form of $M^{n}$ by $\sum_{\alpha, i, j} h_{i j}^{\alpha} w_{i} w_{j} e_{\alpha}$. The square of the length of the second fundamental form is defined by $S=\sum_{\alpha, i, j}\left(h_{i j}^{\alpha}\right)^{2}$.

We call $H=(1 / n)\left(\sum_{\alpha, i} h_{i i}^{\alpha}\right) e_{\alpha}$ the mean curvature vector. $M^{n}$ is said to be a minimal submanifold if $H=0$ and a submanifold with parallel mean curvature if the normalized mean curvature vector is parallel in the normal bundle of $M^{n}$.

The following equality is well known:

$$
R_{i j k l}=\left(\delta_{i k} \delta_{j l}-\delta_{i l} \delta_{j k}\right)+\sum_{\alpha}\left(h_{i k}^{\alpha} h_{j l}^{\alpha}-h_{i l}^{\alpha} h_{j k}^{\alpha}\right)
$$

where $R_{i j k l}$ are the components of the curvature tensor of $M^{n}$.

The following two lemmas are basic for our aim.

Received by the editors June 26, 1984.

1980 Mathematics Subject Classification. Primary 53C40; Secondary 53C20.

${ }^{1}$ The author was supported by the Max-Planck-Institut für Mathematik, Sonderforschungsbereich 40 of the University of Bonn. 
LEMMA 1. Let $M$ be a complete Riemannian manifold whose sectional curvature is bounded from below, and let $f$ be a nonnegative $\mathbf{C}^{2}$-function on $M$ bounded from above. If there exists a positive constant $c$ such that $\Delta f \geqslant c f$ holds, where $\Delta f$ is the Laplacian of $f$, then $f=0$.

Proof. Otherwise, for a point $p, f(p)>0$. According to Omori's Theorem $\mathrm{A}^{\prime}$ in [3], there exists another point $q$ such that $f(q) \geqslant f(p)>0$ and $\Delta f(q)<c f(p)$. On the other hand, $\Delta f(q) \geqslant c f(q) \geqslant c f(p)$. This is a contradiction.

LEMMA 2. Let $M^{n}$ be an $n$-dimensional submanifold in the sphere $S^{n+p}$. If the square of the length of the second fundamental form of $M^{n}$ is bounded from above, then the sectional curvature of $M^{n}$ is bounded from below.

Proof. Let $X=\sum_{i} \lambda^{i} e_{i}, Y=\sum_{i} \mu^{i} e_{i}$ be any two orthogonal unit vectors of $T_{p}\left(M^{n}\right)$. From (1) the sectional curvature determined by $X$ and $Y$ is equal to

$$
\begin{aligned}
R_{i j k l} \lambda^{i} \mu^{j} \lambda^{k} \mu^{\prime}= & \sum_{i}\left(\lambda^{i}\right)^{2} \sum_{j}\left(\mu^{j}\right)^{2}-\left(\sum_{i} \lambda^{i} \mu^{i}\right)^{2} \\
& +\sum_{\alpha, i, j, k, l}\left(h_{i k}^{\alpha} \lambda^{i} \lambda^{k}\right)\left(h_{j l}^{\alpha} \mu^{j} \mu^{\prime}\right)-\sum_{\alpha}\left(\sum_{i, j} h_{i j}^{\alpha} \lambda^{i} \mu^{j}\right)^{2} \\
= & 1+\sum_{\alpha, i, j, k, l}\left(h_{i k}^{\alpha} \lambda^{i} \lambda^{k}\right)\left(h_{j l}^{\alpha} \mu^{j} \mu^{\prime}\right)-\sum_{\alpha}\left(\sum_{i, j} h_{i j}^{\alpha} \lambda^{i} \mu^{j}\right)^{2} .
\end{aligned}
$$

For fixed $\alpha$ we choose orthonormal frames such that $h_{i j}^{\alpha} a_{i}^{\alpha} \delta_{i j}$. Thus,

$$
\left(\sum_{i, j} h_{i j}^{\alpha} \lambda^{i} \mu^{j}\right)^{2}=\left(\sum_{i} a_{i}^{\alpha} \lambda^{i} \mu^{i}\right)^{2} \leqslant n S
$$

and

$$
\sum_{i, j, k, l}\left(h_{i k}^{\alpha} \lambda^{i} \lambda^{k}\right)\left(h_{j l}^{\alpha} \mu^{j} \mu^{l}\right)=\sum_{i, j} a_{i}^{\alpha} a_{j}^{\alpha}\left(\lambda^{i} \mu^{j}\right)^{2}
$$

Since $\sum_{i}\left(a_{i}^{\alpha}\right)^{2} \leqslant S$, we have

$$
a_{i}^{\alpha} a_{j}^{\alpha} \geqslant-\frac{1}{2}\left(\left(a_{i}^{\alpha}\right)^{2}+\left(a_{j}^{\alpha}\right)^{2}\right) \geqslant-S / 2 .
$$

Thus, we obtain from (4) and (5),

$$
\begin{aligned}
\sum_{i, j, k, l}\left(h_{i k}^{\alpha} \lambda^{i} \lambda^{k}\right)\left(h_{j l}^{\alpha} \mu^{j} \mu^{l}\right) & \geqslant \sum_{i \neq j} a_{i}^{\alpha} a_{j}^{\alpha}\left(\lambda^{i} \mu^{j}\right)^{2} \geqslant-\frac{S}{2} \sum_{i \neq j}\left(\lambda^{i} \mu^{j}\right)^{2} \\
& \geqslant-\frac{S}{2} \frac{n(n-1)}{2}
\end{aligned}
$$

Noting that the estimations (3) and (6) hold for each index $\alpha$, we conclude

$$
R_{i j k l} \lambda^{i} \mu^{j} \lambda^{k} \mu^{l} \geqslant 1-C S,
$$

where $C$ is a positive constant. The lemma follows from (7). 
2. Results. Now we can state our result as follows.

THEOREM 1. Let $M^{n}$ be an $n$-dimensional complete minimal submanifold in the sphere $S^{n+p}$. If $S \leqslant n /(2-1 / p)-\varepsilon$ for a positive constant $\varepsilon$, then $M^{n}$ is totally geodesic.

Proof. In this case, referring to [4, 5.31 and 5.32, p. 93], we have

$$
\Delta S \geqslant S(n-(2-1 / p) S) \geqslant(2-1 / p) \varepsilon S,
$$

and the sectional curvature of $M^{n}$ is bounded from below by the assumption and Lemma 2. Applying Lemma 1, we complete the proof.

Referring to the corresponding proofs in [5] and using our lemmas in the same way as above, we can easily obtain the following theorems, which are proved by S. T. Yau in the compact case.

THEOREM 2. Let $M^{n}$ be a complete minimal submanifold in the sphere $S^{n+p}$. Suppose the sectional curvature of $M^{n}$ is everywhere not less than $(p-1) /(2 p-1)+\varepsilon$ for $a$ positive constant $\varepsilon$. Then $M^{n}$ is totally geodesic.

Proof. Since the sectional curvature of $M^{n}$ is bounded from below and $M^{n}$ is minimal, we deduce from $R=n(n-1)-S$ that $S$ is bounded from above. The rest of the proof is identical to that of [5, Theorem 15, p. 95].

THEOREM 3. Let $M^{n}$ be a complete minimal submanifold immersed in the sphere $S^{n+p}$. Let $K(x)$ be the function which assigns to each point of $M^{n}$ the infinimum of the sectional curvatures of $M^{n}$ at that point. If $S \geqslant p n(1-2 K)-\varepsilon($ or $S \geqslant p n(1-K)$ $-\varepsilon$ when $K \leqslant 0$ ) for a positive constant $\varepsilon$, then either

(1) $S$ is unbounded from above, or

(2) $M^{n}$ is totally geodesic.

Proof. Refer to [5, Theorem 12, p. 89] and use Lemmas 1 and 2.

THEOREM 4. Let $M^{n}$ be an $n$-dimensional complete submanifold with parallel mean curvature in the sphere $S^{n+p}$ with $p>1$. If $\left(3+n^{1 / 2}-(p-1)^{-1}\right) S \leqslant n-\varepsilon$ for $a$ positive constant $\varepsilon$, then $M^{n}$ lies in a totally geodesic $S^{n+1}$.

Proof. Refer to [5, Theorem 8, p. 78] and use our lemmas.

ACKNOWLEDGEMENT. The author would like to thank the MPI für Mathematik and the SFB 40 of the University of Bonn for their hospitality and also the referee for his helpful suggestions for improving the first version.

\section{REFERENCES}

1. B. Y. Chen, Geometry of submanifolds, Dekker, New York, 1973.

2. T. Hasanis, Characterization of totally umbilical hypersurfaces, Proc. Amer. Math. Soc. 81 (1981), 447-450.

3. H. Omori, Isometric immersions of Riemannian manifolds, J. Math. Soc. Japan 19 (1967), 205-214.

4. J. Simons, Minimal varieties in Riemannian manifolds, Ann. of Math. (2) 88 (1968), 62-105.

5. S. T. Yau, Submanifolds with constant mean curvature, Amer. J. Math. 97 (1975), 76-100.

Max - Planck - Institut fur Mathematik, 5300 Bonn 3, Federal Republic of Germany

Department of Mathematics, Fudan University, Shangai, People's Republic of China 